\section{Two studies of visual search for word targets with controlled rates of presentation*}

\author{
DOUGLAS H. LAWRENCE \\ Stanford University, Stanford, California 94305
}

The two studies reported involve the visual search of word lists for a target item when the rate of presentation is controlled and the words are presented tachistoscopically. In the first study, the target is differentiated physically from the filler items by being capitalized. When the target is the last item in a list, it is readily identified at all presentation rates, but when it is the first word or is embedded in a list, recognition accuracy is inversely related to presentation rate. In the second study, the differentiation between target and filler items is in terms of the presence or absence of category membership. All Ss at all presentation rates do significantly better on lists with an animal word as a target and a set of unrelated words as filler items than on the converse arrangement.

The two exploratory studies reported here stem directly from the work of Neisser and Beller (1965) and from Neisser, Novich, and Lazar (1963) on searching through letter and word lists for a target item. They found that relatively well-trained Ss could scan a column of items, each consisting of a row of six letters, at a rate of approximately 10 items/second when looking for a specified letter target. The error rate, i.e., the failure to detect the target letter, averaged about $23 \%$. The scanning rate was reduced to 6 items/second and the error rate averaged $24 \%$ when the column of items consisted of words three to six letters in length and the target was a word from a designated category, such as animals.

The interest of Neisser and his associates in these tasks was concerned primarily with the information-processing procedures involved. However, the performances observed raise additional interesting questions: (1) What is the maximum rate at which the various types of visual material can be processed, and (2) what are the role of various factors, such as masking, in limiting the rate of information handling? The procedure employed by Neisser is not appropriate for studying these questions, because of the self-paced nature of his task and the possible limit imposed on search rate by the time required for successive saccades and fixations in scanning a vertical list. A recently developed n-channel stroboscopic tachistoscope overcomes these limitations. It permits the use of reasonably long lists of items, controls the presentation rate over a relatively wide range of values, and eliminates the need for successive saccades.

* These studies were supported by Research Grant HD-00897 from the National Institutes of Health.

\section{EXPERIMENT 1}

In the present study the target items are physically differentiated from the filler items; they are capitalized words embedded in a set of lower-case words. This method of physically distinguishing the two types of items was chosen after preliminary experimentation indicated that the differentiating feature must be an integral aspect of the target item in order for $S$ both to detect and to name the target at rapid rates of presentation. The detection of a capitalized target should involve primarily stimulus examination by means of feature extractors, as postulated by Neisser (1967). But, since the $S$ was required to name the word in addition to detecting its presence, more complex processing of the stimulus input is probably involved. The major concern of this study is to determine the influence of the number of filler items preceding and following the target item on recognition accuracy as presentation rate increases. These interdctions between filler and target items are usually conceived of as complex forms of forward and backward masking.

\section{Apparatus}

The n-channel stroboscopic tachistoscope used in these studies has been fully described previously (Lawrence \& Sasaki, 1970). In brief, S faced a vertically oriented black fiberboard surface with a viewing aperture or slot, $11 / 2$ in. long and $3 / 16$ in. wide, cut in it at eye level. His eyes were aligned with this aperture by means of a chinrest $15 \mathrm{in}$. in front of the vertical surface. An item appearing in this aperture was magnified by a rectangular reading glass in the ratio of 1.1:1. A piece of regular bond typing paper, $8 \frac{1}{2} \times 11$ in., was driven vertically behind this aperture at varying rates of speed by means of a Bodine dc motor and Minarik control. Each time the paper moved $1 / 6$ in. (i.e., the distance between two single-spaced lines of type), a strob lamp, which was directly in back of the paper and aligned with the aperture, was fired by means of a photocell detection unit. The strob lamp was part of a Grass photo stimulator (Model PS-2). Because of the brief duration of the flash (less than 50 microseconds), any item appearing in the aperture at that moment appeared to be "stopped." During the off period of the strob lamp, the item moved out of the aperture and the next one was centered in it. Thus, there was no perceived vertical motion of the successive items. The peak intensity of the flash was approximately 750,000 candle power at the half-intensity setting used. This intensity was reduced as a result of the light first passing through the paper and then through a neutral Polaroid filter (HN32X, .010 in.). The room was dark except for a 10-W reading lamp, shielded from $S$ by means of a black wooden tunnel enclosing the front of the apparatus and S's head.

The two main variables are: (1) the flash rate controlled by means of the speed at which the paper moves, and (2) the stimulus rate controlled by means of the spacing between items on the sheet. For instance, if items are single spaced, i.e., $1 / 6$ in. apart, the flash and stimulus rates are equal, and each flash presents a new item. If the items are triple spaced, then the flash rate is three times the stimulus rate because of the two interpolated flashes between items. Thus, these two rates are always partially correlated. In the studies reported here, an attempt was made to keep the flash rate sufficiently high at all stimulus presentation rates so that $S$ would not be distracted by flicker effects. This was done by spacing items further apart on the word lists used at slow presentation rates than on those used at high presentation rates. The actual values used are listed in Table 1.

The range of feasible presentation rates is from 6 to 40 items/second. But in these studies, the maximum rate employed was 20 items/second in order that there would always be at least one interpolated flash between items.

\section{Procedure}

One thousand words, varying in length from three to six letters, were selected from Thorndike and Lorge (1944). An attempt was made to have these

Table 1

Relation of Item Spacing to Presentation Rate Items

per

$6-9$

$10-13$

14-20

Spacing

Quadruple Triple

Double 
representative of the entire list with respect to the relative frequencies of words of these lengths and with respect to the relative frequencies of such words beginning with a given letter of the alphabet. All words were rated as occurring more than three times in a million. This pool of words was entered into a PDP-8 computer so that lists of any length could be drawn. The major restriction was that lists for any given experimental condition could be drawn without replacement. Each list was then typed as a vertical column of words centered on a piece of standard bond paper. One item on each list, the target word, was capitalized; the rest, the filler items, were in lower case.

Control condition (C). This condition was included to determine the adequacy of this device as a simple tachistoscope when only a single capitalized item was presented on a given trial; it also served to familiarize $S$ with the procedure and the nature of the items. Each list or sheet consisted of a single capitalized word. It was positioned at any one of 12 equally-spaced distances from the top of the page. This was done in order to vary the number of flashes that occurred between the beginning of a trial and the presentation of the item. This made it impossible for $S$ to anticipate the exact moment when the item would occur. The item position on successive sheets was determined randomly, with the restriction that each position occur equally frequently in successive blocks of 36 trials. All lists had a row of five dots, or periods, in position zero as a "ready signal." If the target item occurred in the fourth position, for example, this ready signal was followed by a vertical column of three single, equally spaced dots centered on the sheet (cf. Table 1). Thirty-six lists comprised a set, and four sets were compiled. The Ss viewed each set at a different speed or rate of paper movement. These speeds corresponded to presentation rates of 8 , 12,16 , and $20 \mathrm{items} / \mathrm{second}$. But with a single word per sheet and a fixed exposure time, these speeds should have no influence on item recognition, except possibly for slight differences in contrast due to flash rate. Each $S$ received these four speeds in a somewhat different order.

Last word condition $(L W)$. It was anticipated that the greatest interference would come from filler items that follow a target and that when the target item was the last word in a list, recognition accuracy would be minimally influenced by interference from the preceding filler items. To check this assumption and to measure the interference from these items preceding the target, seven sets of 36 lists were constructed in which the target or capitalized word always occurred in the last position. The target item was preceded by 0 through 11 lower-case filler items on various lists, three lists of each type occurring in a set. The first word of eacn set always occupied the position immediately after the ready signal. All words on a list had their first letters vertically aligned. Seven different rates of presentation were used, ranging from 8 to $20 \mathrm{items} /$ second in steps of $2 \mathrm{items} / \mathrm{second}$. All Ss progressed from the slowest to the fastest rate so that practice and rate were completely confounded. The set assigned to a given rate varied between Ss.

First word condition (FW). To determine the influence on recognition accuracy when the target item was followed by varying numbers of filler items, seven sets of 36 lists each were constructed with the target word always the first item on the list. In a given set, the target item occurred equally frequently in the 1st through 12th positions following the ready signal. It was followed by 11 through 0 filler items, i.e., the last item in a list, whether filler or target, always occupied the 12th position. As in the $\mathrm{C}$ condition, if the target item appeared in the 4th position it was preceded by a vertical column of three dots. In all other respects, including presentation rates, this condition was run exactly the same as the LW condition.

Embedded word condition (EW). This condition was concerned with target recognition when there was the possibility of interference effects from filler items both preceding and following the target. Fourteen sets of 36 lists were constructed, each consisting of one target and 11 filler words. In each set, the target word occupied each of the 12 positions in the list equally frequently. The first word in each list immediately followed the ready signal. The presentation rates used were all whole numbers from 7 through 20 items/second. In all other respects, this condition was run the same as the preceding two.

All Ss were run through the four conditions in the order C, LW, FW, and EW. Preliminary work had indicated that this was an increasing order of difficulty. It was hoped that this deliberate confounding of practice and test condition would produce performances at each stage of training characteristic of relatively well-trained Ss. Before testing on a given condition, the nature of the lists involved was described, and each $S$ was run on 10 specially prepared practice lists at the slowest rate used in that condition. Each trial was initiated by E saying "ready." The $S$ placed his chin on the chinrest, focused on the dimly lighted aperture, and then pressed the switch that activated the driving mechanism. The $S$ first saw a brief preexposure field consisting of 10 flashes of light transmitted through the paper. The ready signal, a row of dots, appeared on the 11th flash, and this was followed by the first item in the list at the appropriate interval. There was also a brief postexposure field of varying duration depending upon the spacing of the items on a list. The $S$ was instructed to name the target item and to guess on those trials when he was uncertain. However, some errors were straight omissions. If he failed to detect the target, named a filler item from the list, or guessed a word not on the list, he was told the correct response. This was done in order to ensure that all Ss had equal familiarity with the words in the pool. Occasionally, the words on a list were not centered in the aperture. The $\mathrm{S}$ was instructed to report this, and the trial datum was discarded. This list was rerun at the end of the set. For Condition C, two sets of lists were run each session. In the other conditions, three sets or 108 lists were presented in each 50 min period. Ss were given a brief rest between sets.

The Ss were six paid undergraduate and graduate students, three males and three females. Each had normal or corrected 20/20 vision. Each was run one session a day, 5 days a week.

\section{Results}

The $C$ condition, in which only one item is presented each trial, demonstrates the adequacy of the present device as a simple tachistoscope. Considering all trials at all presentation rates, the average number of errors per $S$ is $1.6 \%$. The range is from $0 \%$ to $4.2 \%$. There is no indication of a correlation between speed of paper movement and errors.

In the LW condition, where the target item is always the last one in a list and is preceded by varying numbers of filler items, there is no evidence that the filler items interfere with the perception of the target word. The average percentage of errors per $S$, over all presentation rates, is only $0.9 \%$. The range is from $0 \%$ to $3.2 \%$. There is no indication of a correlation between number of errors and presentation rate or between error rate and the number of filler items. All Ss reported that the last item in a list produced a clear image that made it easy to perceive the meaning of the word.

In the FW condition, where the target item is the first word in a list and is followed by varying numbers of filler items, accuracy of report is clearly a function of presentation rate. The percentage of errors increases progressively from $1.5 \%$ errors at $8 \mathrm{items} / \mathrm{second}$ to $47.2 \%$ at 20 items/second, as shown in 
Table 2

Percentage Errors for Capitalized Targets

Items per Second

\begin{tabular}{lcccccccccccccc} 
& 7 & 8 & 9 & 10 & 11 & 12 & 13 & 14 & 15 & 16 & 17 & 18 & 19 & 20 \\
\hline FW & - & 1.5 & - & 4.2 & - & 11.6 & - & 25.9 & - & 34.3 & - & 44.9 & - & 47.2 \\
EW* & 2.0 & 7.7 & 9.6 & 16.7 & 17.1 & 18.1 & 24.5 & 25.9 & 22.2 & 29.6 & 35.6 & 34.7 & 39.4 & 41.7 \\
\hline
\end{tabular}

Note-These values are based on all lists including those in which the target item occurred in the last position. Practically no errors were made on the latter.

Table 2. When averaged over all presentation rates, the percentage of errors is independent of the number of filler items involved as long as there is at least one. When the target word is in the 12th position and not followed by a filler item, the percentage of errors is less than $1 \%$ at all presentation rates. The range of individual differences in error scores increases directly with presentation rate; at 20 items/second it is $13.9 \%$ to $86.1 \%$.

The type of error made at the faster presentation rates, i.e., 16, 18 , and 20 items/second, is of considerable interest. Seventy-ight percent of the errors consist of reporting filler items in the list, $19.6 \%$ of words not in the list, and $2.2 \%$ of failures to respond. When a filler item is reported, $88.2 \%$ of the time it is that item immediately following the target item. (If only those trials are considered on which four or more filler items follow the target, the corresponding percentage is $82.2 \%$.) When an error of this type is made, Ss are quite confident that the reported word is the capitalized target word.

In the EW condition, where the target occurs in each position within a list of 12 items, errors increase at high presentation rates (Table 2). (It should be borne in mind that $\mathrm{S}$ had had considerable experience with the procedure and the words by the time he began on the EW lists.) The percentage of errors, averaged over all presentation rates, is independent of target position except when the target occupies the 12th and last position. In the latter case, the percentage of errors is less than $1 \%$ for all rates. Again, the range of individual differences is large and increases with presentation rate; at 20 items/second, it is $16.7 \%$ to $61.0 \%$.

The type of error made at the faster presentation rates, i.e., 16,18 , and 20 items/second, is similar to that found in the FW condition. Seventy-two percent of the errors consist of reporting filler words from the list, $27 \%$ of words not in the list, and $1.0 \%$ of failures to respond. In this condition, errors of the first type can be either items preceding the target (30.9\%) or items following it $(69.1 \%)$. If only the data from lists with the target in Positions 4 through 9 are considered, i.e., those lists on which there are at least three fillers before $(-)$ and after $(+)$ each target, the position of these filler errors with respect to the target item is as follows: -3 or more (9.2\%), -2 (15.8\%), -1 (13.2\%), +1 $(34.0 \%),+2(9.2 \%),+3$ or more $(18.4 \%)$. In the case of errors at -1 and +1 , Ss are quite confident they are reporting a capitalized word. But when the filler item reported is more removed from the target, they are less confident.

When words not on a list are reported, frequently they are related to the target or to the filler items immediately before or after the target, e.g., snout-about. Occasionally, the reported word appears to be a compromise between the target and a filler item, e.g., a target and filler pair of "chain-play" is reported as "plain." These relationships were not further analyzed.

\section{Discussions}

The fastest rate of item processing Neisser and his associates report is about $10 \mathrm{items} / \mathrm{second}$ with an average of $23 \%$ errors when the target is a subset of letters. In the present EW condition, the error rate is only $17 \%$ at a presentation rate of 10 items/second and does not reach $24 \%$ until the rate is 13 items/second or greater. The difference between Neisser's studies and those reported here is too great to allow any direct comparison. However, these data at least suggest that the time required for successive saccades in their tasks may place an upper limit on the rate at which items can be processed.

Both physical differentiation of target and filler items, by means of capitalized vs lower-case letters in these lists, and relatively high flash intensity were employed to make the search task relatively easy. Insofar as this was achieved, this differentiation of target and filler items undoubtedly minimized forward and backward masking effects and other possible interactions between successive items. Consequently, it is not too surprising that when the target word is the last item in a list, as in the LW and EW conditions, it is as readily perceived as it is in the control condition. Neither the number of preceding filler items nor the rate of presentation is related to recognition accuracy. On the other hand, the presence of one or more filler items following the target interferes with recognition, and this effect increases directly with rate of presentation. The obvious suggestion is that this is due to some form of backward masking.

If we follow the suggestions made by Neisser (1967), an alternative approach is to assume that the FW task requires $S$ to engage in two relatively distinct and successive operations. He first must detect the stimulus features characteristic of the target. (In the FW condition, this may involve only the detecting of the presence of the first word in the list.) This is the signal for the second operation, which is to process the stimulus input to a level where the word can be recognized and reported. Assuming, first, that the detection operation takes time and, second, that the two operations occur in succession, it then becomes more probable, as the presentation rate is increased, that the input from the target will have been replaced by the input from the following filler item before the detection operation is complete (cf. Potter \& Levy, 1969). As a consequence, $S$ processes this filler item to the level of recognition. Implicit in this account is the further assumption that the information in the stimulus that triggers the detection operation is different from that required for the recognition operation.

The tentative explanation offered for errors in the FW condition also seems appropriate for the EW condition, if additional assumptions are made. Assuming that there is some probability that the detection operation can be activated by characteristics of the filler items, $S$ may report that item or a following one even though this occurs before the capitalized item appears. An altemative is that $S$ processes some of the filler items that occur prior to the target item even in the absence of any detection operation. If such an act of recognition is completed just as the stimulus features of the target occur, the latter are ascribed to the recognized filler item and result in an anticipatory error. But as $S$ is set to inhibit word recognition until the stimulus characteristics for detection occur, the predominant error tends to be an item following the target.

\section{EXPERIMENT 2}

The second study is concerned with the difficulty of recognizing target items in the EW condition when there is no physical differentiation between the target and filler items, and differentiation of targets from fillers is in terms of category membership. The targets are a set of animal words and the fillers are a set of unrelated words, similar to the design employed by Neisser and Beller (1965). In addition, performance on this task is contrasted with that on the converse arrangement in which 
Table 3

Percentnge Error for Categorized and Noncategorized Targets

\begin{tabular}{lcccccccccc}
\hline & Items/Second & 6 & 7 & 8 & 9 & 11 & 13 & 15 & 17 & 19 \\
\hline A & Odd Ss & 10.0 & 14.8 & 17.2 & 19.6 & 24.8 & 36.2 & 44.4 & 48.4 & 51.6 \\
Lists & New Ss & 24.8 & 22.8 & 29.6 & 30.8 & 33.2 & 41.2 & 56.4 & 62.0 & 64.4 \\
NA & Old Ss & 25.2 & 32.0 & 35.6 & 40.4 & 51.2 & 50.8 & 56.8 & 59.6 & 66.0 \\
Lists & New Ss & 35.2 & 43.2 & 36.8 & 40.0 & 46.4 & 58.8 & 67.8 & 69.2 & 70.8 \\
\hline
\end{tabular}

the targets are nonanimal words embedded in a list of filler items all of which are animal words.

\section{Procedure}

One hundred animal names, including mammals, birds, and fishes, were compiled. They varied from three to six letters in length. All were familiar animal names, as indicated by preliminary testing on undergraduate students. It was apparent, however, that the distribution of word lengths, the relative frequency of first and last letters in these words, and a number of other aspects were not representative of a randomly selected group of words. If used as target items in a list of randomly selected nonanimal words, it was conceivable that $S$ might be able to detect the target occasionally on the basis of its unique stimulus features independently of any need to recognize it on the basis of its category membership. Consequently, a set of 100 nonanimal words was compiled that matched the animal words in most stimulus features such as letter length, relative frequency of first letter, and the like. In selecting the nonanimal words, the following rules were employed; no word was used if changing, adding, or subtracting one letter converted it into an animal word.

Two types of lists were constructed, the animal lists (A), containing 1 animal word as the target and 10 nonanimal words as fillers, and the nonanimal lists (NA), containing 1 nonanimal word as target and 10 animal words as fillers. The fillers for each list were randomly selected, with the restriction that no filler item occur twice on the same list. The target word was chosen at random without replacement until the appropriate pool of words was exhausted. In each of the two types of lists, the position occupied by the target was determined randomly. Fifty lists constituted a set, and nine sets of each type were constructed. All words were typed in lower-case letters. As in the previous study, a row of dots occurred in Position 0 and served as a ready signal.

The presentation rates used were $6,7,8$, $9,11,13,15,17$, and 19 items/second. The spacing of items on a list for a given presentation rate is shown in Table 1. At a given rate, each $S$ had one set of a given type, but the set used differed between Ss. Ail Ss started at the slowest rate and progressed to the fastest so that practice and rate were completely confounded. Sessions were $50 \mathrm{~min}$ long, during which each $\mathbf{S}$ had two sets of lists. Each was given at the same presentation rate, but one was the $A$ and the other the NA type. Their order of occurrence varied from session to session.

As preliminary training, each $S$ was fully informed about the nature of the task and required to read aloud once each of the 100 animal and 100 nonanimal terms. He then received 20 practice trials at the slowest rate of presentation, 10 with each type of list. If $S$ made an error at any time during training, either by failing to report or by reporting the wrong word, he was told the correct target item so that all Ss would have comparable familiarity with them.

The 10 Ss used in this study were paid graduate and undergraduate students, three males and seven females. Five of them had been Ss in Experiment 1 (old Ss) and five were naive with respect to this type of task (new Ss). Each S had normal or corrected $20 / 20$ vision.

\section{Results}

The percentage of errors averaged over five $\mathrm{Ss}$ as a function of presentation rate for each type of list is shown in Table 3. In interpreting these functions, it should be kept in mind that the confounding of practice and presentation rate probably increased the error level at the slower rates. All Ss were quite unfamiliar with the target lists at that stage of practice.

The most notable effect in Table 3 is the considerably greater accuracy on the $A$ than on the NA lists. Considering percentage of errors summed over all presentation rates, each $S$ does better on the former lists than on the latter. The same is true at each presentation rate for at least 9 of the 10 Ss.

The five old Ss, who had participated in Experiment 1, are consistently better on the $\mathbf{A}$ lists than are the five new Ss. A two-way analysis of variance (Type of S by Presentation Rate) indicates that the two groups are significantly different ( $1 \%$ level) in terms of average error score. There is no evidence of a significant interaction between groups and presentation rate. A similar analysis of the NA data fails to indicate any consistent difference between the groups. The individual differences are large for both types of lists at the faster presentation rates. At 19 items/second, the range is from $36 \%$ to $70 \%$ on the A task and from $50 \%$ to $82 \%$ on the NA task.

Ss rarely make the error of reporting a filler item from a given list. Knowing the category membership of the target item, they presumably suppress any tendency to report a perceived item that does not fit this requirement. The distribution of other errors, i.e., combined omissions and words belonging to the same category as the target, with respect to target position within a list is shown in Table 4. These values were computed by summing for each $S$ the total errors made for all presentation rates when the target occupied a given position, dividing by the total number of times the target had. occurred in that position, and then averaging across Ss.

For both groups and both types of lists, a target in the last position on a list is easily identified. Targets in the first two positions, while somewhat harder to identify, are clearly easier than are targets in the remaining eight positions. This same pattern is found when only the data from the four fastest presentation rates are considered. With presentation rates of 15 items/second and above, there is a clear trend in both types of lists for the error rate to be inversely related to target length, presumably due to the vertical alignment of the first letters in all words, which results in a lower probability that the end letter in the longer words are masked.

\section{Discussions}

In the Neisser and Beller study (1965), well-practiced Ss achieved a scanning rate of 6 items/second with a $24 \%$ error rate when scanning down a column of nonanimal filler words in search of an animal target. In the present study with controlled presentation rates, the group of new Ss, who are relatively unfamiliar with both the targets and the procedures, show a comparable error rate at 6 items/second. However, the group of old Ss, who are very familiar with the procedures, but only slightly familiar with the target items, do not obtain this error rate until the presentation rate has increased to 11 items/second. Assuming the latter group is more comparable to the Ss trained in the Neisser and Beller study, the suggestion is that the need for successive saccades in their study may have limited the rate at which material of this sort can be processed. This tentative conclusion needs to be tempered, however, in view of the gross differences between the two studies.

Of more interest is the clear difference in difficulty of the $A$ and NA lists at all presentation rates and for all Ss. In one sense, there is less uncertainty in the pool 
Table 4

Percentage Error by Target Position in Experiment 2

Target Position

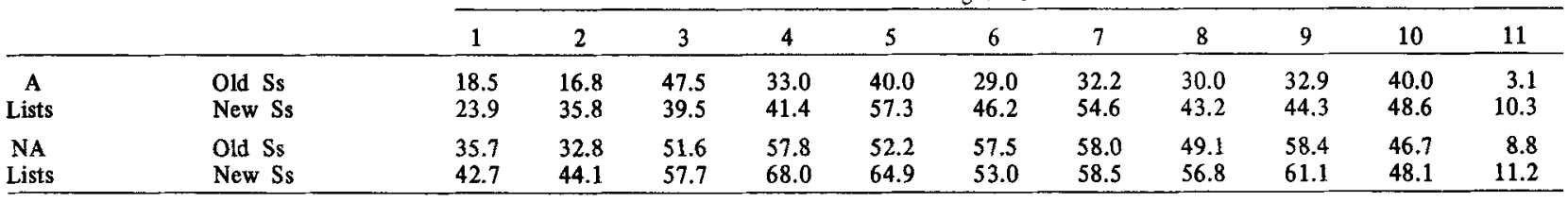

of animal words than in the pool of nonanimal words. It is highly probably that if a $S$ were asked to generate 100 animal and 100 nonanimal items, all between three and six letters in length, there would be more overlap with the items used in this study in the former case than in the latter. Consequently, once $S$ is set to look for animal targets in a list, many of the specific targets actually used would be readily available for retrieval, even though $S$ had not memorized the target items. This would not be the case when S was set to look for non animal targets. This differential availability of the two types of targets could well account for the performance differences observed, especially at the slower presentation rates while Ss are becoming familiar with the target items,

This account in terms of uncertainty differences stresses the availability of the target items in memory. A supplementary, but more speculative, account emphasizes the role of target detection. With both types of lists, $S$ is relatively unfamiliar with the target items. His ability to recognize a target item hinges on his discrimination of animal from nonanimal words, i.e., a difference in category membership rather than in physical characteristics. It is assumed that in the A-type lists, $S$ can be set for this characteristic. As a result, only those items possessing this characteristic are processed to the level of recognition; filler items are processed minimally and readily rejected. In the case of the NA-type lists, where the filler items have a common characteristic and the target item can be identified only by its absence, all items must be processed to the same degree before the decision to reject or accept can be made. The basic assumption is that there is an asymmetry between the case in which a definite characteristic can be used as a signal for further processing and the case in which only the absence of such a characteristic can be used in this way. The selective mechanism proposed is analogous to the feature extractors discussed by Neisser (1967), but it is assumed that is operates at the level of category relationships rather than at the level of stimulus features.

The efficacy of this proposed selective detection mechanism cannot be tested by the present data because of the differential uncertainty in the two target sets. However, if the differential uncertainty in the two sets of targets could be removed, perhaps by having $S$ thoroughly memorize both sets so that they were equally available in memory (at least by any conventional test of availability), the same facilitation of performance on the A lists relative to the NA lists should be found if this selective detection mechanism were operating. Furthermore, the advantage of the A lists should disappear if the memorized animal targets were embedded in a list of filler items consisting of unmemorized animal words. There would be no basis on which the selective detection mechanism could discriminate between target and filler items. Similarly, if the memorized nonanimal targets were embedded in a list of unmemorized, nonanimal filler items, recognition performance should be no worse than that on the present NA task. Even though in the present NA case there is a clear basis for discrimination between filler and target items in terms of category membership, the fact that the targets can be detected only by the absence of the category relationship entails that all items would have to be processed equally just as in the case where no differentiation is involved. The results of a recent experiment by Sasaki (1970), in which these conditions were used, support these predictions.

\section{REFERENCES}

LAWRENCE, D. H., \& SASAKI, E. H. An n-channel stroboscopic tachistoscope for typewritten stimuli. Behavior Research Methods \& Instrumentation, 1970, 2, 63-65.

NEISSER, U. Cognitive psychology. New York: Appleton-Century-Crofts, 1967.

NEISSER, U., \& BELLER, H. K. Searching through word lists. British Joumal of Psychology, 1965, 56, 349-358.

NEISSER, U., NOVICK, R., \& LAZAR, R. Searching for ten targets simultaneously. Perceptual \& Motor Skills, 1963, 17, 955-961. POTTER, M. C., \& LEVY, E. I. Recognition memory for a rapid sequence of pictures. Journal of Experimental Psychology, 1969, $81,10-15$.

SASAKI, E. H. The influence of number and type of targets upon rapid scanning of word lists. Unpublished $\mathrm{PhD}$ dissertation, Stanford University, 1970.

THORNDIKE, E. L., \& LORGE, I. The teacher's word book of 30,000 words. New York: Bureau of Publications, Teachers College, Columbia University, 1944.

(Accepted for publication July 6, 1970.) 